\title{
Baryonic inflow and outflow histories in disk galaxies as revealed from observations of distant star-forming galaxies
}

\author{
Daisuke Toyouchi and Masashi Chiba \\ Astronomical Institute, Tohoku University, Aoba-ku, Sendai 980-8578, Japan \\ email: toyouchi@astr.tohoku.ac.jp \\ email: chiba@astr.tohoku.ac.jp
}

\begin{abstract}
Gas inflow and outflow are the most important processes, which determine the structural and chemical evolution of a disk galaxy like the Milky Way. In order to get new insights into these baryonic processes in Milky Way like galaxies (MWLGs), we consider the data of distant star-forming galaxies and investigate the evolution of the radial density profile of their stellar components and the associated total amount of gaseous inflow and outflow. For this purpose, we analyze the redshift evolution of their stellar mass distribution, combined with the scaling relations between the mass of baryonic components, star formation rate and chemical abundance for both high- and low- $z$ star-forming galaxies. As a result, we find the new relations between star formation rate and inflow/outflow rate as deduced from these distant galaxies, which will provide fundamental information for understanding the structural and chemical evolution of MWLGs.
\end{abstract}

Keywords. Disk galaxies, Gas inflow and outflow, Structural and chemical evolution

\section{Our method}

We investigate the time evolution of the surface density profiles of gas inflow and outflow rates in MWLGs, denoted as $\Sigma_{\text {in }}$ and $\Sigma_{\text {out }}$, respectively. For this purpose, we solve the mass conservation equations for gas and heavy elements at any time, $t$, and radius, $R$, along the disk. We then calculate $\Sigma_{\text {in }}(t, R)$ and $\Sigma_{\text {out }}(t, R)$ given the time evolutions of the radial profiles of surface mass densities of gas, stellar and heavy elements. For these later information in MWLGs, we adopt the recent observational results for distant star-forming galaxies, including the growth of the stellar mass distribution based on the abundance matching method (van Dokkum et al. 2013), their scaling relation between the mass of baryonic components, star formation rate and chemical abundance (Mannucci et al. 2010), as well as the supposed evolution of their radial metallicity gradients (VilaCostas \& Edmunds 1992; Stott et al. 2014). Thus, we derive the gas inflow and outflow histories in MWLGs as revealed from the observations of distant star-forming galaxies (see Toyouchi \& Chiba 2015 for details).

\section{New insights into galactic gas inflow and outflow processes}

Based on this method, we obtain the radial profiles of $\Sigma_{\text {in }}$ and $\Sigma_{\text {out }}$, both of which are higher at smaller $R$ at all epochs, and find that their central values monotonically decline with decreasing redshifts. We also investigate the dependences of $\Sigma_{\text {in }}(t, R)$ and $\Sigma_{\text {out }}(t, R)$ on a star formation rate density, $\Sigma_{\mathrm{SFR}}(t, R)$. This procedure allows us to inspect whether the assumption adopted in many previous studies, namely that inflow and outflow rates in galaxies are proportional to their SFR, is indeed valid or not. Figure 

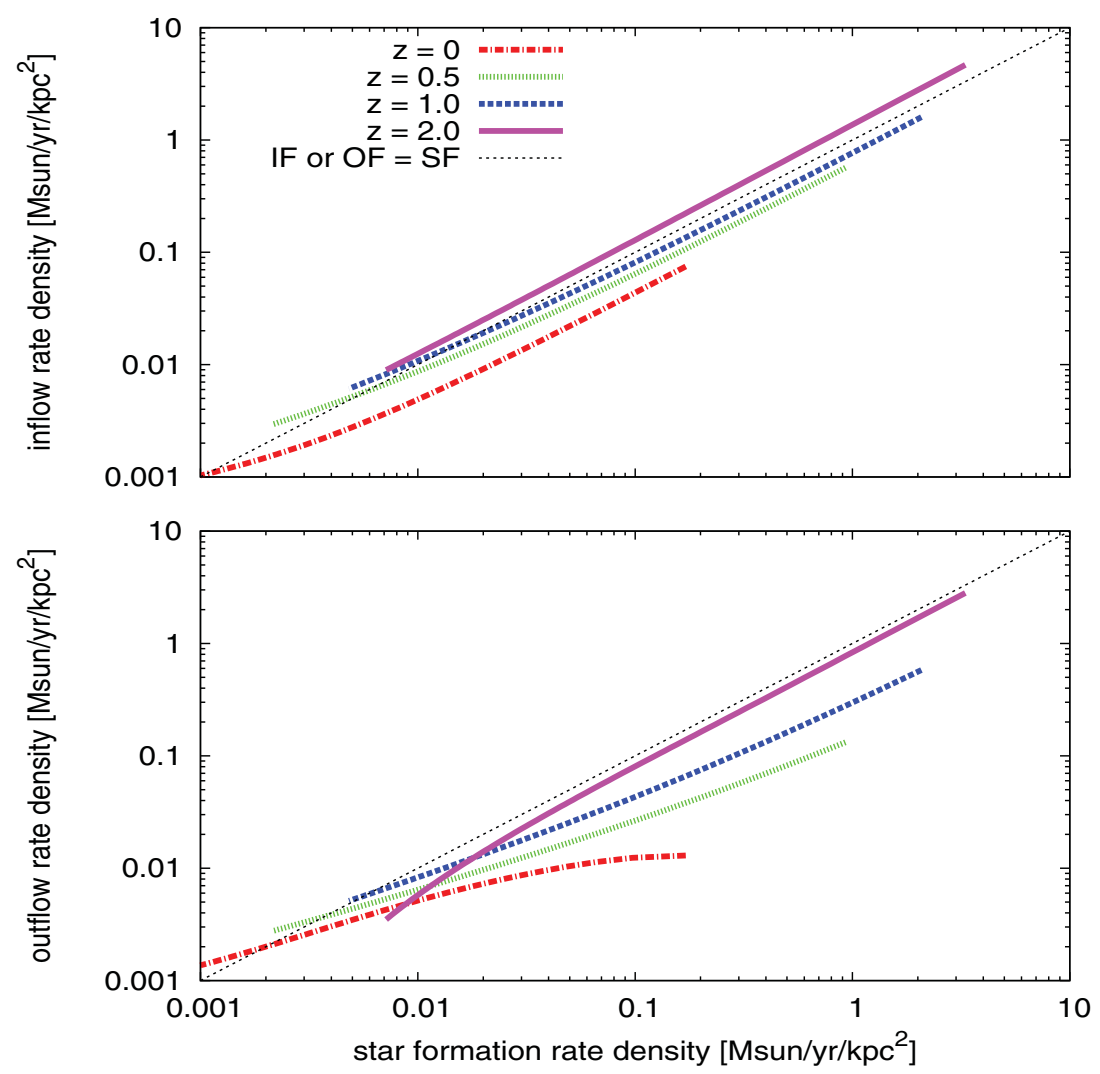

Figure 1. The inflow and outflow rate densities (top and bottom panels, respectively) as a function of the star formation rate density at redshifts of $z=0,0.5,1$ and 2 .

1 shows $\Sigma_{\text {in }}$ and $\Sigma_{\text {out }}$ as a function of $\Sigma_{\text {SFR }}$ at redshifts of $0,0.5,1.0$ and 2.0. Thin dotted lines show $\Sigma_{\mathrm{in}}=\Sigma_{\mathrm{SFR}}$ (top panel) and $\Sigma_{\text {out }}=\Sigma_{\mathrm{SFR}}$ (bottom panel) for comparison. We find that although $\Sigma_{\text {in }}$ is always approximately proportional to $\Sigma_{\mathrm{SFR}}$, such a proportional relation is not necessarily satisfied for $\Sigma_{\text {out }}$. This result for outflow may imply that the assumption of proportionality of outflow rate to star formation rate is simplistic. We also find that these properties of outflow rate can be simply understood in the framework of momentum-driven or energy-driven wind mechanism. To conclude, this work allows us to distinguish the main driving mechanism for galactic outflows, which can significantly influence the structural and chemical evolution of star-forming galaxies.

\section{References}

Mannucci, F., Cresci, G., Maiolino, R., Marconi, A., \& Gnerucci, A. 2010, MNRAS, 408, 2115

Stott, J. P., Sobral, D., Swinbank, A. M., et al. 2014, MNRAS, 443, 2695

van Dokkum, P. G., Leja, J., Nelson, E. J., et al. 2013, ApJL, 771, L35

Vila-Costas, M. B. \& Edmunds, M. G. 1992, MNRAS, 259, 121 